\title{
Patterns of prescription, hospitalizations and costs of herpes zoster in patients at risk, from a large Italian claims database
}

\author{
Silvia Calabria ${ }^{1}$, Giulia Ronconi ${ }^{1}$, Letizia Dondi ${ }^{1}$, Carlo Piccinni $^{1}$, Antonella Pedrini ${ }^{1}$, Immacolata Esposito $^{2}$, Pierluigi Viale ${ }^{3}$, \\ Nello Martini ${ }^{1}$ \\ ${ }^{1}$ Fondazione ReS (Ricerca e Salute) - Research and Health Foundation, Casalecchio di Reno, Bologna - Italy \\ ${ }^{2}$ Drugs \& Health Srl, Rome - Italy \\ ${ }^{3}$ Infectious Disease Unit, Department of Medical and Surgical Sciences, Teaching Hospital S. Orsola-Malpighi, Alma Mater Studiorum Univer- \\ sity of Bologna, Bologna - Italy
}

\begin{abstract}
Purpose: This observational study aimed to investigate the incidence of herpes zoster (HZ) among at-risk subjects aged $\geq 50$ years, characterize them and assess annual healthcare utilization and costs from the Italian National Health System (NHS) perspective.

Methods: Records of reimbursed drug prescriptions, hospitalizations and outpatient specialist care from the Fondazione ReS database were linked to identify patients aged $\geq 50$ years at $\mathrm{HZ}$ risk (i.e. cardiovascular disease/ chronic obstructive pulmonary disease/diabetes/immunosuppression, according to the Italian National Vaccine Prevention Plan - PNPV 2017-2019) in 2013. New HZ events (incidence per 1,000) were researched in 2 years, and subjects with $\mathrm{HZ}$ in the previous year were excluded. Antiviral and pain therapy consumptions, hospitalizations for $\mathrm{HZ}$ and costs paid by NHS were assessed annually.

Results: From $12,562,609$ inhabitants in 2013 , a total of $1,004,705$ patients ( $18.5 \%$ aged $\geq 50$ years) at risk without a previous event were selected. The 2-year incidence of HZ was 5.9 per 1,000 (mean age $74 \pm 10$ years; $54.3 \%$ female). Patients aged $80-89$ ( 7.2 per 1,000), females $(6.7$ per 1,000$)$ and immunosuppressed subjects ( 6.9 per $1,000)$ had the highest incidence rates. One year after the new $\mathrm{HZ}$ episode, $82.2 \%$ were treated with specific antivirals (79.3\% brivudine), generating an annual average cost/treated of $€ 106 ; 8.0 \%$ were hospitalized for $\mathrm{HZ}$, with an average cost/hospitalized of $€ 3,927$; the overall mean cost/incident patient was $€ 402$.

Conclusions: This analysis provided $\mathrm{HZ}$ incidence in subjects aged $\geq 50$ years considered at risk by the PNPV and its burden from the NHS perspective. Our findings can help health governance to improve clinical decisions and economic positioning concerning zoster vaccine plan.
\end{abstract}

Keywords: Big data, Healthcare costs, Herpes zoster virus, Public health practice, Zoster vaccine

\section{Introduction}

Herpes zoster (HZ), also known as 'shingles', is a common and debilitating disorder caused by reactivation of varicella zoster virus (VZV), which is typically contracted during

Received: February 10, 2020

Accepted: July 27, 2020

Published online: September 10, 2020

Corresponding author:

Silvia Calabria

Fondazione ReS (Ricerca e Salute) - Research and Health Foundation Via Magnanelli 6/3 - 40033, Casalecchio di Reno

Bologna - Italy

calabria@fondazioneres.it childhood. Following the primary infection, which manifests clinically as chickenpox, the virus becomes latent in the sensory cranial nerve and spinal ganglia and may reactivate, giving rise to nervous and skin symptoms with a dermatomal distribution typical of $\mathrm{HZ}(1)$.

In Europe, it is estimated that more than 1.7 million people suffer from $\mathrm{HZ}$. The estimated overall mean incidence is about 3.4-4.8 per 1,000 person-years. The risk of $\mathrm{HZ}$ episodes increases with age, up to more than 11 per 1,000 personyears among 80-year-old subjects (2). Several medical conditions may increase the risk of $\mathrm{HZ}$ occurrence or induce symptom worsening. Age and immunosuppression seem to be the major risk factors, but also other conditions, including diabetes mellitus, cardiovascular (CV) disease and chronic obstructive pulmonary disease (COPD), have been shown to be associated with an increased risk of $\mathrm{HZ}$ (3-7). The Italian National Vaccine Prevention Plan (PNPV) 2017-2019 
presented the new timetable for the vaccinations recommended and reimbursed by the Italian National Health System (NHS), by age and risk groups. Among the novelties, the zoster vaccine has been introduced for people aged 65 and older, and for subjects $\geq 50$ years with at least a disease that may increase the risk of $\mathrm{HZ}$ or cause symptom worsening (CV disease, COPD, diabetes and immunosuppression) (4). Immunization can reduce both $\mathrm{HZ}$ incidence and complication rates, including ocular and postherpetic neuralgia (PHN)-related pain (estimated in approximately $20 \%$ of cases of $\mathrm{HZ})(4,8,9)$.

This observational study, based on real-world data, aimed at identifying the incidence of $\mathrm{HZ}$ in patients aged 50 and older with at least one disease that increases the risk of $\mathrm{HZ}$, according to the last PNPV. Additional purposes of this study were to characterize the $\mathrm{HZ}$ incident subjects and to determine their annual healthcare resource utilization and costs from the NHS perspective.

\section{Methods}

\section{Data source}

This observational analysis was performed using the Fondazione ReS (Ricerca e Salute - Research and Health Foundation) database. In collaboration with CINECA (Interuniversity Consortium in Bologna), the Fondazione ReS database routinely collects and integrates complete and available NHS administrative databases of demographics, free filled drug prescriptions, hospital discharges and outpatient specialist services. Data are completely anonymized at the source for each single patient. Therefore, the ReS database comprises inhabitants residing in different Italian regions. The data source has completeness and high usefulness, as demonstrated by several studies in the international literature (10-12). Pharmaceutical data consist of all free-filled drug prescriptions, for which the following information was recorded: active substances (according to the Anatomical Therapeutic Chemical [ATC] classification), medicinal products (by means of the authorization number issued by the Italian Medicines Agency [AIFA] for commercialization), dose, number of packages, dispensing date and costs reimbursed by the NHS. Hospital discharge data collect diagnoses and procedures, according to the Italian adjustment of the ninth version of the International Classification of Diseases - Clinical Modification (ICD-9-CM) (13). The Italian ambulatory care database collects invasive/non-invasive and diagnostic specialist procedures according to the national classification system. Demographics were anonymous, according to the Italian data protection and privacy law. Ethical approval was not sought for the present study because it was based on the reuse of anonymous administrative data and conducted for institutional purposes, in agreement with the Italian health facilities (regions and local health units [LHUs]).

\section{Cohort selection and follow-up}

From 1 January to 31 December 2013 (accrual period), patients aged $\geq 50$ years with at least one risk factor for $\mathrm{HZ}$ according to the PNPV 2017-2019 (i.e. CV disease, COPD, diabetes, immunosuppression) were selected ( $\mathrm{HZ}$ at-risk cohort). The conditions at risk for $\mathrm{HZ}$ were searched through algorithms based on specific administrative databases (all used codes are detailed in Supplemental Table 1, Selection criteria of patients at risk of herpes zoster in the accrual period (2013), by risk factor, available online). The date of the first event identifying patients at risk was considered as the index date since the $\mathrm{HZ}$ events were searched. During the 2 years after this index date, subjects who were prescribed brivudine, the uniquely approved and recommended antiviral in Italy against $\mathrm{HZ}$ infection (ATC code: J05AB15), and/or those admitted to hospital due to a primary/secondary diagnosis of HZ (ICD-9-CM code: 053.x) were considered patients with a zoster episode. Moreover, in order to select solely incident patients, those who experienced a $\mathrm{HZ}$ event during the year before the $\mathrm{HZ}$ occurrence were excluded, adopting the same criteria as described above. The incident cohort was analysed in terms of consumption and mean cost per patient treated $(€)$ of specific treatments during two different follow-up periods, starting from the $\mathrm{HZ}$ event. Prescriptions of specific antivirals (i.e. brivudine, acyclovir, valacyclovir and famcyclovir) were evaluated over 1 year, whereas recommended systemic pain therapies (i.e. pregabalin, gabapentin and tramadol) (7) were evaluated over the first 6 months.

Moreover, hospitalizations with a primary diagnosis of HZ (ICD-9-CM code: 053.x) were searched over 1 year from the $\mathrm{HZ}$ event and analysed as the percentage of hospitalized patients, mean length of stay (days) and mean expenditure per patient.

Lastly, for each $\mathrm{HZ}$ incident patient that could be followed for 1 year from the zoster occurrence, the annual costs to the NHS were assessed. Specifically, pharmaceutical costs were extrapolated by the gross expenditure of local pharmacy sales and the real hospital price (inclusive of value-added tax [VAT]) of hospital pharmacy supplies. The in-hospital expenses were derived from the DRG (Diagnosis-Related Group) system tariffs.

\section{Additional investigation}

In order to fulfil the real-world practice information needs about $\mathrm{HZ}$, an additional investigation was performed by comparing the 2-year incidence rates between the $\mathrm{HZ}$ at-risk cohort (cases) and the ones not at risk (controls), matched 1:1 for gender, age and LHU of residency. The comparison was provided by age and gender. Annual mean overall expenditures per patient are also shown for both cohorts.

\section{Statistical analyses}

The $\mathrm{HZ}$ at-risk cohort was described by gender, age, type and number of risk factors. The 2-year incidence of $\mathrm{HZ}$ episodes (per 1,000 at-risk patients aged $\geq 50$ years) was also estimated for each risk factor subgroup. The 2 -year incidence rates of each subgroup were compared with z-test, using the group with the highest incidence rate as reference; a $p$-value $<0.05$ was considered statistically significant.

The $\mathrm{HZ}$ incident cohort was analysed by using descriptive statistics: categorical variables were reported as frequencies and continuous variables as means. 
One-year costs, both for the whole cohort and for every risk factor group, are shown as mean euro per capita and percentage on the integrated expenditure.

\section{Results}

Within the ReS database, 12,562,609 Italian inhabitants had complete data of their healthcare services provided by the NHS from 1 January 2012 to 31 December 2015. The ReS database consists of administrative data accurately representative of the Italian population. Indeed, distributions by age and gender are consistent with those provided by the Italian Institute of Statistics for 2013 (Supplemental Figure 1, Percentage distribution of the 2013 Italian population by age group, from the ReS database and from the Italian Institute of Statistics (ISTAT), available online), and the results provided by this observational analysis reflect the real demographic characteristics of candidates to $\mathrm{HZ}$ vaccination.

Among patients aged 50 and older ( $n=5,419,586)$, $1,004,705$ (18.5\%) had at least one risk factor for $\mathrm{HZ}$ during 2013 and were not hospitalized for $\mathrm{HZ}$ and/or were not prescribed brivudine over the previous year. In particular, $87.1 \%$, $11.9 \%, 1.0 \%$ and $0.08 \%$ of subjects had one, two, three and four risk factors, respectively. Specifically, 58.9\% of patients were affected by diabetes, $29.5 \%$ by CV disease, $22.7 \%$ by COPD and $2.8 \%$ by immunosuppression (Fig. 1). Certainly, a clinical assessment is necessary in order to confirm and integrate comorbidity cohorts (e.g. administrative databases do not identify patients with diabetes treated only with diet) as

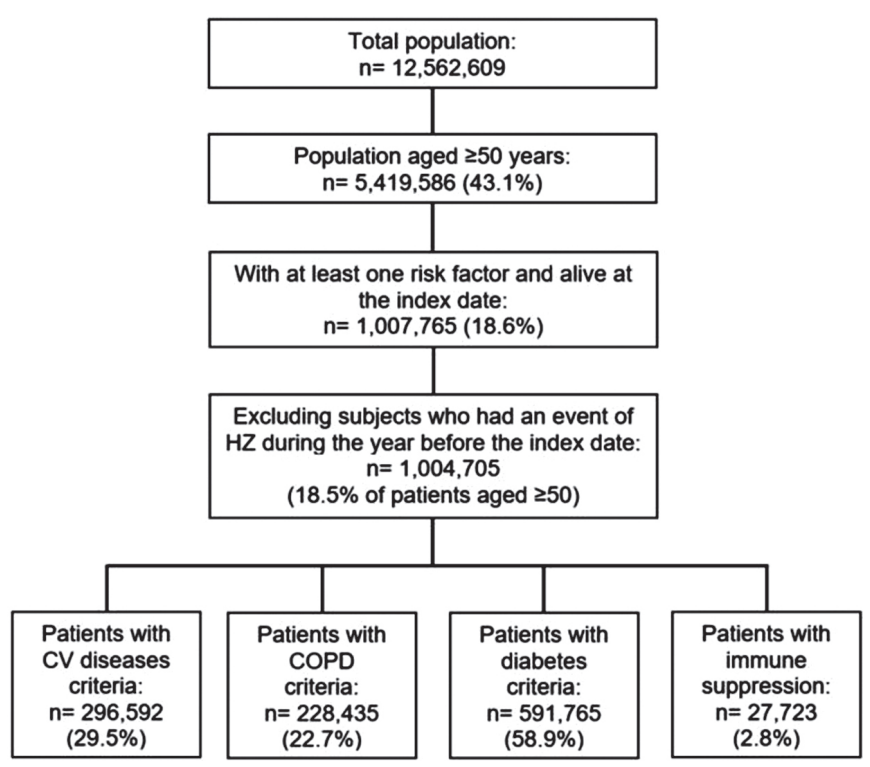

Fig. 1 - Selection of the patients aged $\geq 50$ years with risk factors from the ReS database. The flowchart depicts the selection of patients aged $\geq 50$ years at risk for herpes zoster and eligible for the zoster vaccine according to the Italian National Vaccine Prevention Plan 2017-2019. The sum of the comorbidity groups is higher than the overall $\mathrm{HZ}$ at-risk cohort, because patients could have more than one risk factor.

$\mathrm{HZ}$ = herpes zoster; ReS = Ricerca e Salute; $\mathrm{CV}$ = cardiovascular; COPD = chronic obstructive pulmonary disease. well as to define the severity of target clinical conditions and of related comorbidities. Over the 2-year follow-up, 5,916 patients of the $\mathrm{HZ}$ at-risk cohort experienced $\mathrm{HZ}$, resulting in a 2-year incidence rate of 5.9 per 1,000. We are fully aware of the limitations of administrative data analyses and of the possibility of underestimating the incidence of $\mathrm{HZ}$. That is why we wanted to make sure that our $\mathrm{HZ}$ incidence findings were as much aligned as possible with reality. Nevertheless, the calculated annual incidence was 2.9 per 1,000 person-years among individuals aged $\geq 50$ years at risk for $\mathrm{HZ}$. This is about a half of the 2-year rate; therefore, this is what we expected by considering a minimal loss to follow-up. Although this 1-year incidence rate is not directly comparable with findings from other studies, probably due to differences in both study designs and populations analysed, it appears similar to the estimates across the world (3.0-5.0 per 1,000 person-years), Europe (2.0-4.6 per 1,000 person-years) and Italy (1.59-6.9 per 1,000 persons) $(4,7,14,15)$, recorded among patients $\geq 50$ years with comorbidities (16).

Specifically, $92.1 \%$ of subjects were identified through brivudine prescriptions, $7.1 \%$ through hospitalizations for $\mathrm{HZ}$ (primary/secondary diagnosis) and $0.8 \%$ by both criteria. Since $\mathrm{HZ}$ is typically diagnosed and treated in the outpatient setting, hospitalizations represent the most severe cases. The lack of general practitioners' (GPs) visits and their associated diagnoses in the ReS database could be considered a limitation of this study, but hospital prescriptions of brivudine are very few, and therefore this criterion could reliably identify the $\mathrm{HZ}$ cases diagnosed by GPs. Certainly, the $\mathrm{HZ}$ cases among outpatients could be underestimated as they could be treated with other antivirals (i.e. acyclovir, valacyclovir and famcyclovir) not included in the selection criteria. Indeed, they could not have been specific for $\mathrm{HZ}$ cases, because their reimbursement circumstances (Nota AIFA 84 (17)) also include some herpes simplex virus and VZV conditions.

The mean age ( \pm standard deviation) of patients with a new $\mathrm{HZ}$ event was $74 \pm 10$ years and $54.3 \%$ were female (Tab. I). The 2-year incidence increased with age, reaching the highest values in the 80- to 89-year-old group (7.2 per $1,000)$ and among females $(6.7$ per 1,000$)$, consistently with the literature $(6,7,18)$. Within risk factor groups, the 2-year incidence rates were, in ascending order: 5.6/1,000 among diabetics, 6.1/1,000 in patients with CV disease, 6.8/1,000 in patients with COPD and 6.9/1,000 in patients with weakened immune system. In accordance with the literature, immunosuppressed patients experienced the highest incidence $(6.9 / 1,000$ vs. $5.6 / 1,000$ in diabetics: $p<0.01)$. This suggests that the vaccine could provide the support needed to the immune system to prevent the expression of $\mathrm{HZ}(6,16)$. The forthcoming non-live recombinant subunit vaccine could fulfil this need, as to date it is not contraindicated in immunocompromised subjects. Moreover, although the 2-year incidence rate increased with the number of risk factors from $5.9 / 1,000(n=1)$ to $12.2 / 1,000(n=4)$, it was independent of them $(p>0.05)$.

Specific healthcare consumption and costs were assessed for 5,455 patients $(92.2 \%$ of the incident cohort) who could be followed up for 1 year.

In order to evaluate the recurrent use of antivirals after a new diagnosis of $\mathrm{HZ}$, the 1-year rate of patients treated with 
TABLE I - Epidemiological characterization of the cohort of patients aged $\geq 50$ years and with a new event of $\mathrm{HZ}$

\begin{tabular}{|c|c|c|c|}
\hline & $\begin{array}{c}\text { Patients with } \\
\text { a new event } \\
\text { of } \mathrm{HZ} \\
(\mathrm{n}=5,916)\end{array}$ & $\begin{array}{l}\text { Incidence rate } \\
\text { (per } 1,000 \text { at- } \\
\text { risk patients } \\
\text { aged } \geq 50 \text { ) }\end{array}$ & p-value \\
\hline \multicolumn{4}{|l|}{ Age (y) } \\
\hline Mean \pm SD & $74 \pm 10$ & & \\
\hline Median & 75 & & \\
\hline \multicolumn{4}{|l|}{ Age group (y), n (\%) } \\
\hline $50-59$ & $597(10.0)$ & 4.0 & Ref \\
\hline $60-69$ & $1,347(22.8)$ & 5.1 & $<0.01$ \\
\hline $70-79$ & $2,110(35.7)$ & 6.4 & $<0.01$ \\
\hline $80-89$ & $1,595(27.0)$ & 7.2 & $<0.01$ \\
\hline$\geq 90$ & $267(4.5)$ & 6.8 & $<0.01$ \\
\hline \multicolumn{4}{|l|}{ Gender } \\
\hline Female & $3,210(54.3)$ & 6.7 & Ref \\
\hline Male & $2,706(45.7)$ & 5.1 & $<0.01$ \\
\hline \multicolumn{4}{|c|}{ Distribution of risk factors, $n(\%)$} \\
\hline CV disease & $1,814(30.6)$ & 6.1 & 0.13 \\
\hline COPD & $1,553(26.2)$ & 6.8 & 0.92 \\
\hline Diabetes & $3,319(56.1)$ & 5.6 & $<0.01$ \\
\hline Immunosuppression & $190(3.2)$ & 6.9 & Ref \\
\hline \multicolumn{4}{|c|}{ No. of risk factors, $n$ (\%) } \\
\hline 1 & $5,137(86.8)$ & 5.9 & 0.45 \\
\hline 2 & $716(12.1)$ & 6.0 & 0.46 \\
\hline 3 & $62(1.05)$ & 6.4 & 0.51 \\
\hline 4 & $1(0.02)$ & 12.2 & Ref \\
\hline
\end{tabular}

The population eligible for the zoster vaccine, according to the Italian National Vaccine Prevention Plan 2017-2019, is described by age, gender, percentage distribution and number of risk factors. The 2-year incidence of $\mathrm{HZ}$ is shown by age, gender and risk factor. Results of the z-test between incident rates of each subgroup are provided.

$\mathrm{COPD}=$ chronic obstructive pulmonary disease; $\mathrm{CV}=$ cardiovascular; $\mathrm{HZ}=$ herpes zoster; $\mathrm{SD}=$ standard deviation.

at least one antiviral drug among brivudine, acyclovir, valacyclovir and famcyclovir was analysed (Tab. II), with $82.2 \%$ of patients who were not lost to 1-year follow-up. On average, each patient costed yearly €106 to the NHS (acyclovir and valacyclovir having the lowest cost) and received 1.4 boxes (from 1.1 of brivudine to 3.7 of acyclovir). According to risk factors, the mean annual cost per treated patient ranged from a minimum of $€ 105$ for diabetics to a maximum of $€ 115$ for immunosuppressed patients.

Literature data on medical outpatient care costs show discrepancies (14). By evaluating only the supply of antivirals, this study found a 1-year cost for specific medicines that accounted for $21.6 \%$ of the total expenditure for a patient experiencing $\mathrm{HZ}$ in the presence of risk factors, in the perspective of the NHS. This is in line with the proportion of patients treated with antiviral therapies found by a Dutch study, and with the costs reported in European studies ranging from $€ 118$ to $€ 242$ regardless of age $(14,16)$.

Another specific analysis aimed to evaluate the use of recommended systemic pain therapies (pregabalin, gabapentin and tramadol) during the first 6 months after the $\mathrm{HZ}$ episode, identifying 1,435 patients treated with at least one pain drug (Tab. II). On average, each patient costed $€ 94$ to the NHS and received 4.6 boxes of pain drugs during the 6 -month follow-up. Since there is poor consensus on the definition of postherpetic pain duration $(2,6,15,16,18,19)$, which may occur until 1 month (acute pain), from 30 to 90 days (subacute pain) or from 3 to 12 months (PHN) from diagnosis, the choice to search for these pain therapies over a 6-month period after the new $\mathrm{HZ}$ event could have generated both an overestimation and an underestimation of the complications' severity. Moreover, the use of pharmaceuticals rather than hospitalization codes for PHN could also have underestimated its burden.

This real-world data analysis allowed to assess only the expenses reimbursed by the NHS. An underestimation of all the direct costs of patients with $\mathrm{HZ}$ was inevitable, because administrative databases do not consider the private outpatient care. The last official report on the Italian pharmaceutical expenditure estimated that $28 \%$ of the mean per capita expenses for drugs in 2018 are paid by the patient (20). In addition, subjects who deny the use of specific drugs reimbursed by the NHS should also be taken into account. The limitations of the healthcare resource availability from the administrative databases can be an obstacle for decision makers to evaluate the real-world evidence (RWE) (21).

The 1-year rate of hospitalizations with a primary diagnosis of $\mathrm{HZ}$, with or without complications (ICD-9-CM code 053.x) of incident patients not lost to follow-up ( $n=5,455)$, was $8.0 \%$. They were hospitalized for an average of 16.6 days and 1.1 times (Tab. II). The mean annual cost of a patient hospitalized due to $\mathrm{HZ}$ was $€ 3,927$. If risk factors are considered, immunosuppressed patients were admitted to hospital more frequently (16.8\%) than the others, resulting also in the most expensive subjects, with an average cost of $€ 5,172$ per hospitalized patient. In contrast, patients with diabetes were less frequently hospitalized (6.0\%) and patients with COPD had the lowest hospitalization costs (€3,550 per patient). In our analysis, the 1-year hospitalization rate due to $\mathrm{HZ}$ and the mean length of stay seem higher compared to those reported in other studies $(16,22)$. Nevertheless, although this analysis did not distinguish between hospitalizations for $\mathrm{HZ}$ and those for HZ-related complications (e.g. PHN, zoster ophthalmicus), this strategy was grounded on the fact that the use of the primary diagnosis with the ICD-9-CM code 053.x, including $\mathrm{HZ}$ and its complications, can be highly predictive of an episode of $\mathrm{HZ}$, especially in the older adult population (22). Moreover, the longer length of stay can be due to the restriction of the study cohort to subjects with comorbidities, such as CV disease or COPD. Although there are some discrepancies in estimating the NHS absorption of the expenses of hospitalizations due to $\mathrm{HZ}$ and its complications, the annual mean inpatient care cost is consistent with that of Panatto et al (14). 
TABLE II - Specific healthcare consumptions and costs of incident patients

\begin{tabular}{|c|c|c|c|c|c|c|c|c|c|}
\hline \multirow[t]{2}{*}{ Risk factor groups } & \multicolumn{3}{|c|}{$\begin{array}{l}\text { Patients treated with antivirals* } \\
\qquad(n=4,483)\end{array}$} & \multicolumn{3}{|c|}{$\begin{array}{c}\text { Patients treated with pain } \\
\text { therapies }{ }^{\dagger}(n=1,435)\end{array}$} & \multicolumn{3}{|c|}{$\begin{array}{l}\text { Patients hospitalized due to } \mathrm{HZ}^{*} \\
\qquad(\mathrm{n}=437)\end{array}$} \\
\hline & $\begin{array}{c}\% \\
\text { treated } \\
\text { in group }\end{array}$ & $\begin{array}{c}\text { Mean } \\
\text { expenditure/ } \\
\text { treated } \\
\text { patient }(€)\end{array}$ & $\begin{array}{c}\text { Mean } \\
\text { boxes/ } \\
\text { treated } \\
\text { patient (n) }\end{array}$ & $\begin{array}{c}\% \\
\text { treated } \\
\text { in group }\end{array}$ & $\begin{array}{c}\text { Mean } \\
\text { expenditure/ } \\
\text { treated } \\
\text { patient }(€)\end{array}$ & $\begin{array}{c}\text { Mean } \\
\text { boxes/ } \\
\text { treated } \\
\text { patient }(n)\end{array}$ & $\begin{array}{c}\% \\
\text { hospitalized } \\
\text { in group }\end{array}$ & $\begin{array}{c}\text { Mean } \\
\text { expenditure/ } \\
\text { hospitalized } \\
\text { patient }(€)\end{array}$ & $\begin{array}{l}\text { Mean } \\
\text { length } \\
\text { of stay } \\
\text { (d) }\end{array}$ \\
\hline CV disease & 90.1 & 107 & 1.4 & 26.7 & 85 & 4.5 & 9.6 & 4,205 & 16.7 \\
\hline COPD & 76.9 & 106 & 1.4 & 26.0 & 103 & 5.4 & 8.8 & 3,550 & 18.4 \\
\hline Diabetes & 80.8 & 105 & 1.3 & 26.4 & 101 & 4.7 & 6.0 & 4,028 & 18.8 \\
\hline Immunosuppression & 74.9 & 115 & 2.2 & 37.7 & 155 & 6.6 & 16.8 & 5,172 & 16.0 \\
\hline Total $(n=5,455)$ & 82.2 & 106 & 1.4 & 26.3 & 94 & 4.6 & 8.0 & 3,927 & 16.6 \\
\hline
\end{tabular}

In subjects with 1-year data available $(n=5,455)$, percentage distribution of patients treated with antivirals (acyclovir, valacyclovir and famcyclovir) or systemic pain therapies (gabapentin, pregabalin and tramadol) and of patients admitted to hospital due to HZ are shown, by risk factor and overall. Drug consumption is provided as mean number of boxes per patient treated, while hospitalizations are presented as mean length of stay (days). The costs of all healthcare services are shown as average per patient treated/hospitalized.

$\mathrm{CV}=$ cardiovascular; $\mathrm{COPD}=$ chronic obstructive pulmonary disease; $\mathrm{HZ}$ = herpes zoster.

*At 1-year follow-up.

${ }^{+}$At 6-month follow-up.

TABLE III - Comparison of 2-year incidence rates between patients at risk and not at risk and integrated costs

\begin{tabular}{lccc}
\hline & $\begin{array}{c}\text { Incidence rate } \\
\text { (per 1,000) } \\
\text { among at-risk } \\
\text { patients } \\
\text { (n= 5,916) }\end{array}$ & $\begin{array}{c}\text { Incidence rate } \\
\text { (per 1,000) } \\
\text { among not at- } \\
\text { risk patients } \\
\text { (n = 4,764) }\end{array}$ & \\
\hline Overall & 5.9 & 4.7 & $<0.01$ \\
\hline Age group (y) & & 2.9 & $<0.01$ \\
$50-59$ & 4.0 & 4.3 & $<0.01$ \\
$60-69$ & 5.1 & 5.2 & $<0.01$ \\
$70-79$ & 6.4 & 5.7 & $<0.01$ \\
$80-89$ & 7.2 & 4.7 & $<0.01$ \\
$\geq 90$ & 6.8 & & \\
\hline Gender & & 3.9 & $<0.01$ \\
Male & 5.1 & 5.6 & $<0.01$ \\
Female & 6.7 & 268 & - \\
Integrated & 402 & & \\
expenditure (€) & & & \\
\hline
\end{tabular}

Results of the z-test that compared the 2-year incidence rates of herpes zoster between patients at risk and not at risk are shown, also by age and gender, taking as reference the group with the highest rate. The integrated expenditure, including pharmaceuticals, hospitalizations and outpatient specialist care, was assessed during the 1-year follow-up and is provided for both cohorts.

In the additional investigation comparing the $\mathrm{HZ}$ at-risk cohort to controls (Tab. III), the difference in the 2-year incidence rates was statistically significant $(5.9 / 1,000$ vs. 4.7/1,000; $\mathrm{p}<.01$ ), confirming this discrepancy also by gender and age.

On average, the NHS yearly spent $€ 402$ for a patient with $\mathrm{HZ}$ or at risk for $\mathrm{HZ}$ vs. $€ 268$ for a patient with $\mathrm{HZ}$ but without the risk factors considered.

\section{Conclusions}

Even if it is not yet included in the routine work for outcomes research of companies, some regulatory agencies (i.e. AIFA for Italy, and Food and Drug Administration for the U.S.A.) use RWE for healthcare decision-making. Differently from randomized clinical trials that are conducted in homogeneous populations, observational studies of real-world settings allow generalizability of the results to the actual population (23). Due to the large size and the representativeness of the Italian population of the ReS database, it was possible to reliably identify and characterize subjects for vaccination against $\mathrm{HZ}$. Analysis of administrative databases can help healthcare stakeholders to study consistently the real burden on the NHS, or rather the costs potentially related to specific diseases and populations. Estimating the size of the burden of individuals eligible for the zoster vaccine will permit to define the impact of vaccination programmes, in terms of health benefits and cost savings. The goal of the PNPV 20172019 was to reach in 2019 a vaccine coverage against $\mathrm{HZ}$ of $50 \%$ among people aged $\geq 65$ years (4). It has been estimated that if the zoster vaccine was extensively administered to the target population, there should be an important decline in the incidence, severity and duration of complications (e.g. ocular and PHN) $(9,24)$. The PNPV recommends the zoster vaccine to immunocompetent people aged 65 and older and to subjects from the age of 50 with risk factors, including the immunocompromised state, without a recent history of $H Z$. Considering an incidence rate of 6.3 per 1,000 (2005) in people aged $\geq 50$ years, the PNPV reported that in Italy the economic burden of $\mathrm{HZ}$ and $\mathrm{PHN}$ could be estimated at $€ 41.2$ million, including both direct (two-thirds of the total expenditure, about 28 million) and indirect costs (4). With the aim of reaching $50 \%$ of vaccine coverage in 2018 , the saving has been estimated at $€ 13,868,063$. This estimation will tend to increase every year because the number of new patients vaccinated will be added to patients already immunized against 
the zoster virus. Therefore, also the savings for the State will increase. This real-world observational analysis provided an annual $\mathrm{HZ}$ incidence of 2.9 per 1,000 person-years in subjects aged 50 and older with clinical conditions considered as risk factors, providing a measure of the burden of this condition in the perspective of the NHS. Therefore, our findings can help the health governance to improve clinical decisions and economic positioning concerning the zoster vaccine plan.

\section{Acknowledgements}

The authors thank Paola Luciolli and Luca De Fiore (II Pensiero Scientifico Ed.) for the professional revision of this article.

\section{Disclosures}

Conflict of interest: The authors have nothing to disclose.

Financial support: This research received the unconditional support of GlaxoSmithKline Biologicals SA (study number: 208681), which has not been involved in the collection, analysis and interpretation of data; in the writing of the report; or in the decision to submit the article for publication.

\section{References}

1. Pinchinat $\mathrm{S}$, Cebrian-Cuenca AM, Bricout H, Johnson RW. Similar herpes zoster incidence across Europe: results from a systematic literature review. BMC Infect Dis. 2013;13:170.

2. Johnson RW, Alvarez-Pasquin MJ, Bijl M, et al. Herpes zoster epidemiology, management, and disease and economic burden in Europe: a multidisciplinary perspective. Ther Adv Vaccines. 2015;3(4):109-120.

3. Kawai K, Yawn BP. Risk factors for herpes zoster: a systematic review and meta-analysis. Mayo Clin Proc. 2017;92(12):18061821.

4. Italian Ministry of Health. Piano Nazionale Prevenzione Vaccinale (PNPV) [Italian National Vaccine Prevention Plan] 20172019. Italy 2017; 90.

5. Lai SW, Lin CL, Liao KF. Real-world database investigating the association between diabetes mellitus and herpes zoster in Taiwan. Medicine (Baltimore). 2019;98(18):e15463.

6. Yanni EA, Ferreira G, Guennec M, et al. Burden of herpes zoster in 16 selected immunocompromised populations in England: a cohort study in the Clinical Practice Research Datalink 20002012. BMJ Open. 2018;8(6):e020528.

7. Johnson RW, Rice AS. Clinical practice. Postherpetic neuralgia. N Engl J Med. 2014;371(16):1526-1533.

8. Kang JH, Ho JD, Chen YH, Lin HC. Increased risk of stroke after a herpes zoster attack: a population-based follow-up study. Stroke. 2009;40(11):3443-3448.
9. Gelb LD. Preventing herpes zoster through vaccination. Ophthalmology. 2008;115(2 Suppl):S35-S38.

10. Calabria S, Forcesi E, Dondi L, Pedrini A, Maggioni AP, Martini $\mathrm{N}$. Target population of non-deferrable surgery and uncontrolled severe bleeding related to dabigatran. Cardiovasc Drugs Ther. 2018;32(3):281-286.

11. Cimminiello C, Dondi L, Pedrini A, et al. Patterns of treatment with antiplatelet therapy after an acute coronary syndrome: data from a large database in a community setting. Eur J Prev Cardiol. 2018:2047487318814970.

12. Piccinni C, Ronconi G, Calabria S, et al. Healthcare resources utilisation in primary progressive multiple sclerosis. Neurol Sci. 2018;39(7):1169-1174.

13. Italian Ministry of Work Health and Welfare. Italian version of the International Classification of Diseases - 9th revision Clinical Modification. 2007.

14. Panatto D, Bragazzi NL, Rizzitelli E, et al. Evaluation of the economic burden of Herpes Zoster (HZ) infection. Hum Vaccin Immunother. 2015;11(1):245-262.

15. Kawai K, Gebremeskel BG, Acosta CJ. Systematic review of incidence and complications of herpes zoster: towards a global perspective. BMJ Open. 2014;4(6):e004833.

16. Gialloreti LE, Merito M, Pezzotti P, et al. Epidemiology and economic burden of herpes zoster and post-herpetic neuralgia in Italy: a retrospective, population-based study. BMC Infect Dis. 2010;10:230.

17. Agenzia IdF. Nota 84. https://www.aifa.gov.it/nota-84.

18. Chiyaka ET, Nghiem VT, Zhang L, Deshpande A, Mullen PD, Le P. Cost-effectiveness of herpes zoster vaccination: a systematic review. Pharmacoeconomics. 2019;37(2):169-200.

19. Werner RN, Nikkels AF, Marinovic B, et al. European consensus-based (S2k) guideline on the management of herpes zoster - guided by the European Dermatology Forum (EDF) in cooperation with the European Academy of Dermatology and Venereology (EADV), Part 2: treatment. J Eur Acad Dermatol Venereol. 2017;31(1):20-29.

20. Agenzia IdF. L'uso dei farmaci in Italia. Rapporto Nazionale Anno 2018 [The use of drugs in Italy. National Report Year 2018]. 2018.

21. Malone DC, Brown M, Hurwitz JT, Peters L, Graff JS. Real-world evidence: useful in the real world of US payer decision making? How? When? And what studies? Value Health. 2018;21(3):326333.

22. Jackson LA, Reynolds MA, Harpaz R. Hospitalizations to treat herpes zoster in older adults: causes and validated rates. Clin Infect Dis. 2008;47(6):754-759.

23. Tseng HF, Sy LS. Use of real-world evidence to evaluate the effectiveness of herpes zoster vaccine. J Infect Dis. 2018; 218(suppl_2):S63-S67.

24. Amirthalingam $\mathrm{G}$, Andrews $\mathrm{N}$, Keel $\mathrm{P}$, et al. Evaluation of the effect of the herpes zoster vaccination programme 3 years after its introduction in England: a population-based study. Lancet Public Health. 2018;3(2):e82-e90. 